\title{
Adaptive Course Control System of an Unmanned Surface Vehicle (USV) Based on Back-propagation Neural Network (BPNN)
}

\author{
Yang Fang, Huajun Zhang, Biao Wang \& Chaochao Jiang \\ School of Automation, Wuhan University of Technology, Wuhan 430070, China
}

\begin{abstract}
Unmanned Surface Vehicle (USV) is a small surface naval vessel which navigates and plans by itself. And undoubtedly, it plays a vital role in now and future naval battle and sea rescue. Adaptive course control is a necessary part of USV control, and in recent years, many researchers have done plenty of works on it, however, many of their method did not solve the problem of the accuracy of transfer function or did not solve course maneuvering problems in a simple and efficient way. In view of this problem, we propose a novel adaptive course control method based on back-propagation neural network (BPNN), PID (proportional, integral, derivative) algorithm and stochastic optimization. The method uses model reference adaptive theory and PID algorithm combined together to minimize the course error. And stochastic optimization is also utilized for weight adjustment of neural network. In this way, the system can output rudder angle efficiently and accurately in order to achieve course control of USV. Simulation results showed that the proposed method has great efficiency and theoretical feasibility and its response time is very short.
\end{abstract}

KEYWORD: adaptive course control system; USV; back-propagation neural network; stochastic optimization

\section{INTRODUCTION}

Unmanned Surface Vehicle (USV) is capable of independent planning, autonomous navigation and can complete some small surface tasks like perception of environmental information, target detection, etc. by autonomous mode or artificial intervention. Because of the nonlinear ship motion affected by uncertainty and disturbance of wind, waves, etc., using the traditional identification methods are difficult to obtain an accurate transfer function (model). However, by means of neural networks, fuzzy system to identify ship motion model under various sea conditions, it is possible to accurately reflect the dynamic characteristics. It is true that neural networks have the ability of approaching any nonlinear function precisely, but at the same, lack the ability to generalize, it has seriously affected its accuracy as a reference model. Literature $[1,2]$ use BP algorithm to make online fuzzy neural network controller training in order to achieve true online learning. Since the ship motion model is unknown, BP algorithm cannot accurately calculate the gradient information between the weight and the rudder error, which leads to slow net training and the result is not satisfactory. In aspect of fuzzy adaptive control, literature [3] does systematic research on it, which utilizes the indirect method by fuzzy adaptive gradient method to achieve rapid controller online design, since the study law is uneasy to design, the algorithm cannot completely get rid of the experience factor impact. In order to completely realize the independent design of course controller, lately there have been numerous reports on the use of evolutionary algorithms in online course controller designing. Since genetic algorithm has global search capability, it is most widely used, such as literature $[4,5]$ aim at the learning ability of fuzzy neural network, with minimal rudder error as their goal, genetic algorithm is used to adjust the neural network weights, however, the genetic algorithm optimization time is often too long, therefore in practice, it is difficult to meet the requirements.

In view of these problems, we propose in this paper a novel and simple adaptive course control method based on back-propagation neural network (BPNN) and stochastic optimization, which can swiftly optimize the system which can minimize the error.

\section{BACK-PROPAGATION NEURAL NETWORK (BPNN)}

Back-propagation neural network (BPNN) is used in this paper. A BPNN has an input layer, a hidden layer 
and an output layer. The central idea of BPNN learning algorithm involves back-propagation of errors to change the weights and biases to minimize the total error. BPNNs has self-learning, self-adapting and strong nonlinear mapping ability [6]. BP neural networks have the ability of approaching any nonlinear function precisely, with the help of BP neural network and PID algorithm to train fuzzy neural network controller online in order to achieve true online learning, so as to obtain an accurate transfer function (model). In this paper, a BP Neural Network is used for online training, whose input layer has three neurons (err, derr/dt, Jerr), and output layer has one neuron $(\delta$ (steering angle)). Figure. 1 is the structure of the proposed BPNN.

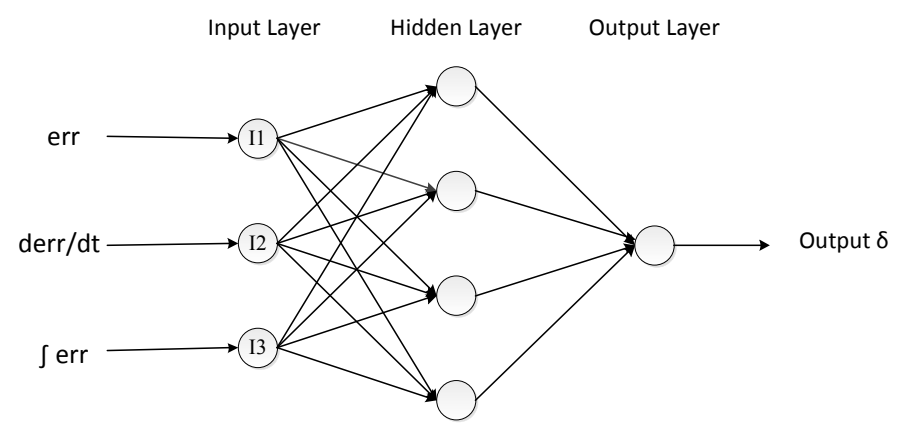

Figure 1 The Structure of the Proposd BPNN

\section{ESTABLISHMENT OF REFERENCE MODEL AND ACTUAL MODEL}

USV, ships sailing in ocean are all rigid motion, through the analysis of the force of the rigid body motion, the ship in the water should meet the following momentum and moment of momentum theorem [7]:

$$
\left\{\begin{array}{c}
\mathbf{m} \dot{\mathbf{U}}+\mathbf{m}(\boldsymbol{\Omega} \times \mathbf{U})=\mathbf{F} \\
\mathbf{J} \dot{\boldsymbol{\Omega}}+\mathbf{\Omega} \times \mathbf{J}=\mathbf{M}
\end{array}\right.
$$

In equation (1), $\mathbf{F}$ represents the resultant force of the ship, M represents the resultant moment of the ship, $\mathbf{m}$ represents the mass of the ship, $\mathbf{J}$ represents the mass moment of inertia, $\dot{\boldsymbol{U}}$ represents the linear displacement acceleration vector and $\dot{\boldsymbol{\Omega}}$ represents the angular displacement acceleration vector, the above vectors are projected in the coordinate system as:

$$
\left\{\begin{array}{c}
\dot{\mathbf{U}}=(\dot{u}, \dot{v}, \dot{w})^{T} \\
\dot{\boldsymbol{\Omega}}=(\dot{p}, \dot{q}, \dot{r})^{T} \\
\mathbf{\Omega} \times \mathbf{U}=(q w-v r) i-(w p-u r) j-(v p-u q) k \\
\boldsymbol{\Omega} \times \mathbf{J}=\left(J_{z}-J_{y}\right) q r i-\left(J_{z}-J_{x}\right) p r j+\left(J_{y}-J_{z}\right) p q k
\end{array}\right.
$$

In equation (2), $J_{x}, J_{y}, J_{z}$ respectively represents the projection of the moment of inertia $\mathbf{J}$ in the coordinate system of the ship. Because of the role of fluid viscous force, added mass inertia force, force on steering wheel, the propeller thrust, complex positive moment and control residual buoyancy moment during the operation of the USV and the ship, make X, Y, Z as the projection of the resultant force in the ship coordinate system, K, M, N as the projection of the resultant moment along the three coordinate axes in the ship coordinate system, so the equation (1) and X, Y, Z, K, M, $\mathrm{N}$ can be substituted into the equation (2) and the space motion equation of the USV and the ship is:

$\left[\begin{array}{c}m \dot{u}+m(q w-v r) \\ m \dot{v}+m(u r-w p) \\ m \dot{w}+m(v p-u q) \\ J_{x} \dot{p}+\left(J_{z}-J_{y}\right) q r \\ J_{y} \dot{q}+\left(J_{x}-J_{z}\right) p r \\ J_{z} \dot{r}+\left(J_{y}-J_{z}\right) p q\end{array}\right]=\left[\begin{array}{c}X \\ Y \\ Z \\ K \\ M \\ N\end{array}\right]$

Equation (3) is the standard six degree of freedom motion equation for the USV.

Take a certain type of USV as an example of the numerical model, the standard six degree of freedom motion equation as described in equation (3) can be sorted into the following matrix equation:

$\left[\begin{array}{c}\dot{u} \\ \dot{v} \\ \dot{w} \\ \dot{p} \\ \dot{q} \\ \dot{r}\end{array}\right]=\mathbf{A}_{w}\left[\begin{array}{c}f_{1}\left(u, v, w, p, q, r, \delta_{r}, \delta_{s}, \delta_{b}, \psi, \phi, \theta\right) \\ f_{2}\left(u, v, w, p, q, r, \delta_{r}, \delta_{s}, \delta_{b}, \psi, \phi, \theta\right) \\ f_{3}\left(u, v, w, p, q, r, \delta_{r}, \delta_{s}, \delta_{b}, \psi, \phi, \theta\right) \\ \vdots \\ f_{50}\left(u, v, w, p, q, r, \delta_{r}, \delta_{s}, \delta_{b}, \psi, \phi, \theta\right) \\ f_{51}\left(u, v, w, p, q, r, \delta_{r}, \delta_{s}, \delta_{b}, \psi, \phi, \theta\right)\end{array}\right]+\left[\begin{array}{c}X_{C} \\ Y_{C} \\ Z_{C} \\ K_{C} \\ M_{C} \\ N_{C}\end{array}\right]$

The state transition relation is as follows:

$$
\begin{aligned}
\dot{\phi} & =p+q \tan \theta \sin \phi+r \tan \theta \cos \phi \\
\dot{\theta} & =q \cos \phi-r \sin \phi \\
\dot{\psi} & =q \frac{\sin \phi}{\cos \theta}+r \frac{\cos \phi}{\cos \theta} \\
\dot{\xi} & =u \cos \psi \cos \theta+v(\cos \psi \sin \theta \sin \phi-\sin \psi \cos \phi)(5) \\
& +w(\cos \psi \sin \theta \cos \phi+\sin \psi \sin \phi) \\
\dot{\eta} & =u \sin \psi \cos \theta+v(\sin \psi \sin \theta \sin \phi+\cos \psi \cos \phi) \\
& +w(\cos \phi \sin \psi \sin \phi-\cos \psi \sin \phi) \\
\dot{\zeta} & =-u \sin \theta+u \cos \theta \sin \phi+w \cos \theta \cos \phi
\end{aligned}
$$

The matrix in equation (4) is the $6 \times 51$ constant coefficient matrix calculated by the parameters of the hull, rudder blade parameters (contains 306 coefficients associated with hull parameters), to a certain type of USV, for example, The heading deflection equation coefficient vector $A_{w}(6,1: 51)$ can be measured by the tank experiment, any steering and static load can be forecast accurately according to $A_{w}$. 
$f_{i}\left(\mathrm{u}, \mathrm{v}, \mathrm{w}, \mathrm{p}, \mathrm{q}, \mathrm{r}, \delta_{r}, \psi, \phi, \theta\right)$ is the function of USV longitudinal velocity $\mathrm{u}$, lateral velocity $\mathrm{v}$ and vertical is to speed $w$, pitch angle velocity $\mathrm{p}$, heeling angle speed $\mathrm{q}$, turn bow angle speed $\mathrm{r}$, rudder angle $\delta_{r}$, heading angle $\psi$, horizontal angle $\phi$ and vertical angle $\theta$.

Vector $\left[\begin{array}{llllll}X_{c} & Y_{c} & Z_{c} & K_{c} & M_{c} & N_{c}\end{array}\right]^{T}$ is the external force and torque for the hull (including the wave force, undercurrent force, rudder force and the corresponding torque). The input of the USV six degree of freedom model as described in equation (4) is the rudder angle, wave force and wave moment, all state can be obtained through integral of equation (4) and (5) during the operation of the USV.

Ultimately, we selected the reference model as:

$G_{m}(\mathrm{~S})=\frac{0.01}{s+0.01}$

The actual model as:

$\mathrm{G}(\mathrm{S})=\frac{1}{s(38 s+1)}$

\section{DESIGN OF THE COURSE CONTROL SYSTEM}

The system uses model reference adaptive theory and stochastic optimization algorithms combined to minimize the course error. And stochastic optimization is also utilized for weight adjustment of neural network. In this way, the system can output rudder angle efficiently and accurately in order to achieve course control of USV. Specifically, first, a course angle enters the system, and then it will be substituted into two algorithms, reference model and BP neural network, for comparison, so the course error is drawn, after that, the error will be optimized to adjust the net weight of neural network by stochastic optimization algorithm, by continuously optimizing neural network, the output rudder angle will generate feedbacks with the input course angle, so that we can achieve the adaptive course control of Unmanned Surface Vehicle (USV). Figure. 2 is the diagram of the course control system.

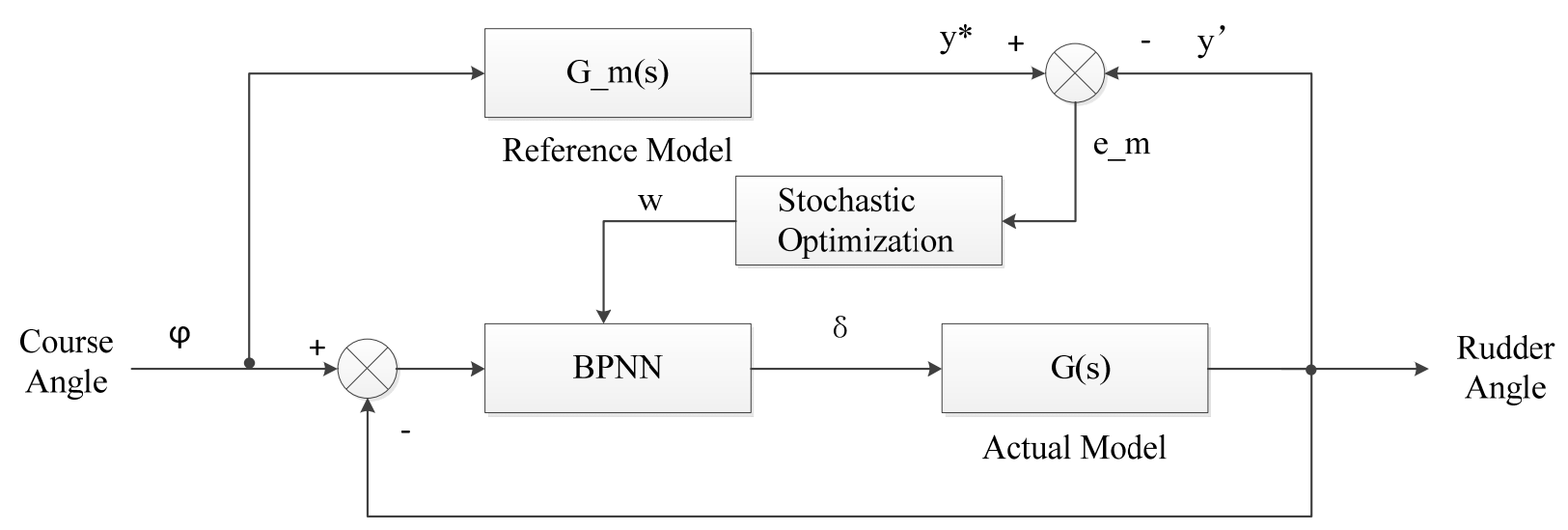

Figure 2 Diagram of the Course Control

The error equation is given below:

err $=\frac{1}{2} e_{m}^{2}$

where $e_{m}=y^{*}-y^{\prime}$.

The goal of our design is to achieve minimal error. And the weight of BP Neural Network is adjusted to meet the goal. Therefore, we seek the partial derivative of err to $\mathrm{w}$. The equation is given below:

$\frac{\partial e r r}{\partial w}=\frac{\partial e r r}{\partial y^{\prime}} \cdot \frac{\partial y^{\prime}}{\partial w}=\frac{\partial e r r}{\partial y^{\prime}} \cdot \frac{\partial y^{\prime}}{\partial \delta} \cdot \frac{\partial \delta}{\partial w}$

Where $\frac{\partial e r r}{\partial y^{\prime}}=-e_{m}$,

$\frac{\partial y \prime}{\partial \delta} \approx 1$, according to stochastic optimization algorithm,

and $\frac{\partial \delta}{\partial w}$ is determined by Back-propagation Neural Network.

\section{RESULTS AND DISCUSSION}

After simulation, we can see that the algorithm proposed is very effective for adaptive course control of USV, and its response time is very short. Obviously, it is a good proof of the theoretical feasibility of this method. Figure 3 is the waveform of the input course angle. Figure 4 is the waveform of the output rudder angle. 


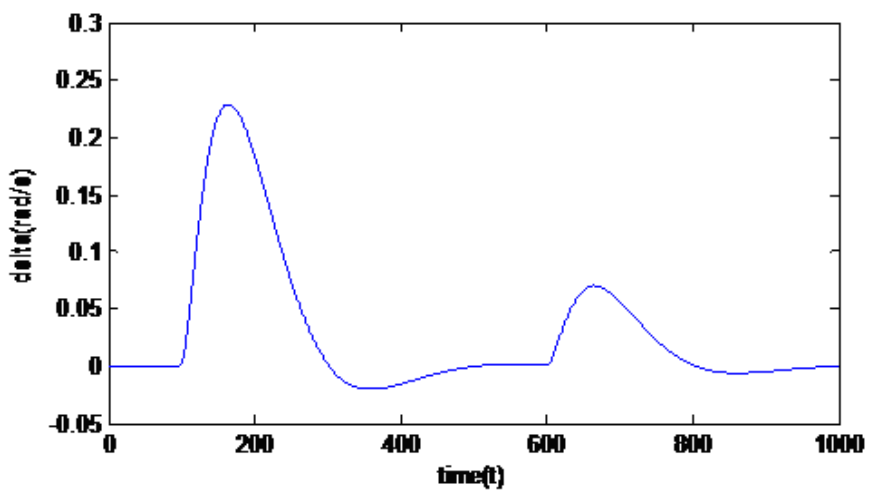

Figure 3 Waveform of the Input Course Angle

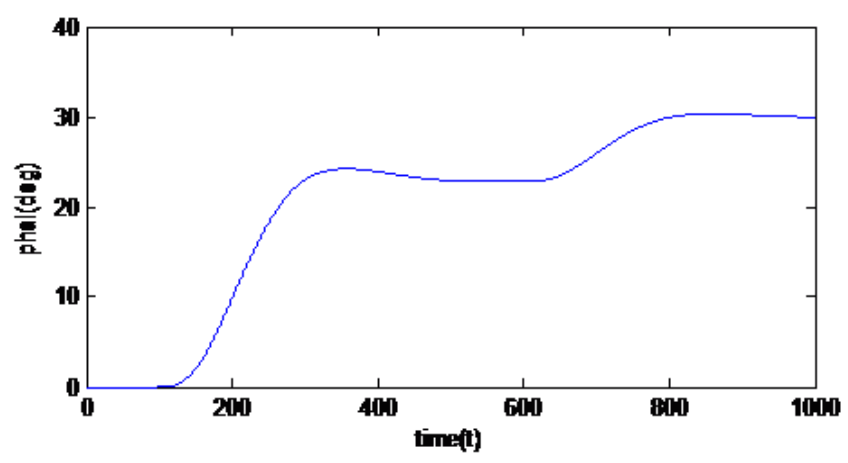

Figure 4 Waveform of the Output Rudder Angle

\section{CONCLUSION}

To achieve adaptive course control of Unmanned Surface Vehicle (USV), this paper focuses on solving the problem at the two following aspects:

1. For the problem of the accuracy of transfer function, since Back-propagation Neural Network has the ability of approaching any nonlinear function precisely, so we utilize Back-propagation Neural Network and PID algorithm for online training of fuzzy neural network controller in order to achieve true online learning, so as to obtain accurate system transfer function ( model).

2. For course maneuvering problems, this paper uses the model reference adaptive herein principle and stochastic optimization algorithm, an extreme dynamic adjustment algorithm for USV control system combined reference model with stochastic optimization algorithm is designed. By estimating the derivative scale factors between objective function and closed-loop system poles, and by simulation of online learning optimization, the optimization simulation results and the conclusion analysis are given. This ensures the strong adaptability of USV in different operating environments, improves the ability of USV's maneuvering control.

\section{ACKNOWLEDGMENTS}

This work was financially supported by Undergraduate program of Independent Innovation Research Fund of Wuhan University of Technology (166811003). The authors are grateful to the editor and anonymous reviewers for their suggestions in improving the quality of the paper.

\section{REFERENCES}

[1] Zhuo Y, Hearn Grant E: Ship Intelligent Autopilot in Narrow Water [C]. Proceeding of the 27th Chinese Control Conference, 2008. 243-248.

[2] Zhuoyong Jiang, Xianglin Fang: Ship Course Online Intelligent Self-learning Control Method [J]. Dalian Maritime University, 2007, 33 (02): 75-79.

[3] Rigatos G, Tzafestas S.: Adaptive Fuzzy Control for the Ship Steering Problem [J]. Mechatronics, 2006, 16(8): 479-489.

[4] Hong Ruanjiu: Fuzzy Neural Network Intelligent Controller Ship Course Nonlinear Systems [J]. Shandong Jiaotong University, 2006, 14 (04): 29-33.

[5] Zhongli Ma, PROJECTILES, Hongda Liu Based on GA-BP Algorithm Intelligent Adaptive Ship Course Control System [J] Ship Engineering, 2006, 28 (05): 23-27.

[6] T. Yahagi, M. Hagiwara and T. Yamaguchi: Neural Network and Fuzzy Signal Processing. Corona Publishing Co., Ltd (1998).

[7] Dianpu Li: Ship Motion and Modeling [M]. Beijing National Defense Industry Press (2008). 